\title{
A NOVEL TOPOLOGY OF EMI FILTER TO SUPPRESS COMMON MODE AND DIFFERENTIAL MODE NOISES OF ELECTRO MAGNETIC INTERFERENCE IN SWITCHING POWER SUPPLIES
}

\author{
P. Ram Mohan ${ }^{1}$, M. Vijaya Kumar ${ }^{2}$ and O.V. Raghava Reddy ${ }^{3}$ \\ ${ }^{1}$ G. Pulla Reddy Engineering, College, Kurnool, Andhra Pradesh, India \\ ${ }^{2}$ JNTU College of Engineering, Anantapur, Andhra Pradesh, India \\ ${ }^{3}$ ISRO Satellite Center, Bangalore, Karnataka, India \\ E-mail: rammohan_cdp@yahoo.co.in
}

\begin{abstract}
This paper deals with Electro Magnetic Interference (EMI) in switching power supplies. The EMI consists of mainly common mode and differential mode noises. Due to interference, the efficiency of the equipment reduces and some times the equipment may be damaged. A procedure to design a novel topology of EMI filter to suppress these two noises is presented in this paper.
\end{abstract}

Keywords: electro magnetic interference, EMI filter, mode, differential, noise, power supplies.

\section{INTRODUCTION}

The demand for power, which has increased tremendously over the last few decades, has forced the power engineers to establish reliable network in order to supply "quality" power to the consumer. Over the years lot of research has been carried out for the supply of quality power to the consumers. This research got a tremendous boost with the strides made in the miniaturization of the electrical industry $[1,2]$. The power electronic devices are very versatile devices capable of delivering power as high as $10 \mathrm{KW}$; also these devices are capable of working at frequencies in the range of hundreds of $\mathrm{KHZ}$ and at the same time the control being only at the gate terminal of the devices, which makes these devices easily controllable $[3,4]$.

Because of these high frequencies EMI occurs. Generally EMI problems arise due to the sudden changes in voltage $(\mathrm{dv} / \mathrm{dt})$ or current $(\mathrm{di} / \mathrm{dt})$ levels in a waveform. In diode rectifier, the line current can be pulse of short duration and the diode recovery current pulse can generate transient voltage spikes in the line inductance. A conductor carrying dv/dt wave acts like an antenna and sensitive signal circuit and appear as noise. The EMI problems create communication line interference with sensitive signal electronic circuits [5-6].

\section{PROBLEM OF EMI}

Widespread use of electric and electronic systems for household, industrial, communications and other applications makes it necessary for circuits to operate on close proximity of each other. Often these circuits affect performance of other nearby circuits adversely via inadvertent coupling of their signals through near and far region, propagating EM fields. This interference is thus called Electro Magnetic Interference (EMI) is emerging to be a major problem for designers. In addition, the use of integrated circuits has reduced the size of electronic equipment; more circuits are being put in less space, thereby increasing the problem of interference.
Equipment designers need to make sure that their equipment will work in the "real world," with other equipment nearby. This implies that the equipment performance should not be affected by external noise sources and equipment should not itself be a source of noise. Avoidance of EMI is a major design objective, besides the principal objective of achieving intended circuit function.

Electromagnetic Compatibility (EMC) is the ability of Electronic Equipment to be able to operate properly despite interference from its Intended Electromagnetic Environment and equally important, not to be a source of undue interference to other equipment intended to working the same environment.

EMI was recognized as a problem almost immediately after commercial use of electrical power became common at the beginning of the 20th century. The problem acquired significant dimensions around 1920 when regular radio transmission worldwide became widespread. The first systematic studies on EMI took place in both the United States and Europe in the 1930s. It was soon recognized that the major sources of EMI were radio transmitters, electrical machinery both in the home and in industry, electrical power lines and automobile ignition. The International Special Committee on Radio Interference (CISPR) held its first meeting in 1934 in Europe.

The last 20 years have seen a virtual explosion in the use of electrically operated devices such as computers, television sets, VCRs, communication equipment such as FAX machines, modems and high speed radio links for digital data transmission. Hand-in-hand with this growth, is the very severe pressure on the electromagnetic spectrum, as the available bands are now crammed very tightly with users of all descriptions. The rapid growth in the use of personal communication systems such as cellular, radios and pagers, and the very large user base for networked systems such as the Internet have 
www.arpnjournals.com

further increased the possibilities of EMI leading to malfunctions.

The magnitude of the problem can be appreciated by considering that if $\mathrm{N}$ is the number of devices that could potentially interfere with each other, the number of interference events would rise as $\mathrm{N}^{\mathrm{N}}$ The growth of all the above systems is continuing at a high pace. Electromagnetic interference or more appropriately electromagnetic pollution is therefore among the most critical issues presently facing the electro-technical community worldwide.

\section{HOW EMI OCCURS}

There are many mechanisms by which EMI reaches a receptor, namely a device that is being affected by it. Some are natural, such as EMI due to cosmic radiation, solar activity or atmospheric lighting discharges. Others are man-made, either intentionally or unintentionally. An example of the former is the interference caused by high voltage power lines or radio transmitters, where the desired electrical operation may interfere with other systems.

Examples of unintentional interference are those caused on the power lines by switching of electrical equipment, or radiation of harmonics from a radio transmitter that may interfere with signals in a band that is in use for another purpose. Obviously, the effects of natural or intentional EMI must be tackled by the EMC characteristics of a system, where it must be designed to operate properly in the presence of such interference.

This requires that typical unintentional EMI source be studied and specified for use by designers. Substantial work has been done on this subject. The responsibility of containing unintentional EMI from devices must clearly lie with the designers of that device. For this, they would need standards on how much EMI is tolerable.

\section{A. Conducted EMI}

When one considers the vast magnitude of interconnected equipment via power lines, telephone lines (including FAX or modems), or networking cables, the potential problems from conducted EMI are truly huge. An example of conducted EMI is given here. The currents $I_{A}$ and $I_{B}$ to two circuits flows through a common source impedance $\mathrm{ZS}$, and also returns via common ground impedance ZG. Thus the net supply voltage to circuit $\mathrm{B}$ is modulated un-intentionally by the current drawn by circuit $A$. This is a very simple example and many other possible mechanisms can be imagined.

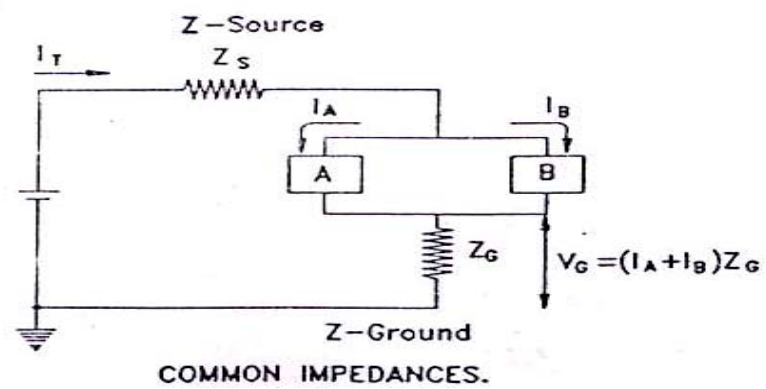

Figure-1. Example of conducted EMI.

It is to be appreciated that actual EMI problems cannot be so easily traced to be solely due radiated or conducted mechanisms, as very often, these noise mechanisms are present simultaneously or transform into each other as they propagate. The example of Conducted EMI is shown in Figure-1.

\section{B. External EMI}

As an example, consider a TV receiver operated in the proximity of a fluorescent tube. It is commonly observed that when the tube light is turned "ON", one sees white lines or spots momentarily superimposed on the TV picture. These are caused by the EMI from contact bounce in switch (or the starter of the tube light) radiating broadband noise which is picked up by the antenna of the TV set. The problem can often be seen even if the TV is operated via a cable connection. In that case travels as induced noise on the mains power line. The example of External EMI is shown in Figure-2.

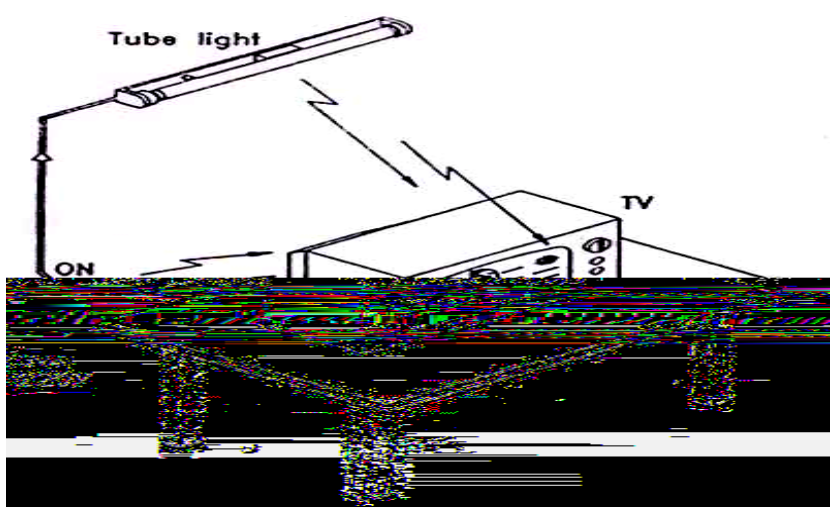

Figure-2. Example of external EMI.

Further more, the same device can both be a source and a receptor of EMI in the above example, the TV was a receptor. A Person can easily perform an experiment where an AM radio set is placed directly over a TV set. Severe disturbance can be picked up in the Radio. Often making it impossible to hear the programme even through the audio from the TV is clear. This is because most modern TV sets contain switched mode power supplies to be able to operate from the mains available around the world. These power supplies radiate substantial noise which disturbs 
www.arpnjournals.com

nearby radios. The TV sound may be clear, because firstly the audio signals are FM and so less susceptible, and the video circuits have been made resistant by appropriate design.

\section{Internal EMI}

The example of the TV given above emphasizes that attention must be given to module-to-module interference within the system because failure to do so may result in the system failing to perform its intended function. As another Example, in an oscilloscope, the magnetic flux from a power transformer can couple to channel amplifier or the high voltage in CRT can couple to amplifier via E field. The example of Internal EMI is shown in Figure-3.

Proper layout, shielding, and cabling are to be used to keep internal EMI within tolerable limits. With the growing complexity of present systems and with concurrent engineering (where different parts of a system are designed simultaneously by multiple design teams) becoming more popular to reduce development cycle times to meet tough market demands; prediction and management of internal EMI is a very important aspect of electronics design.

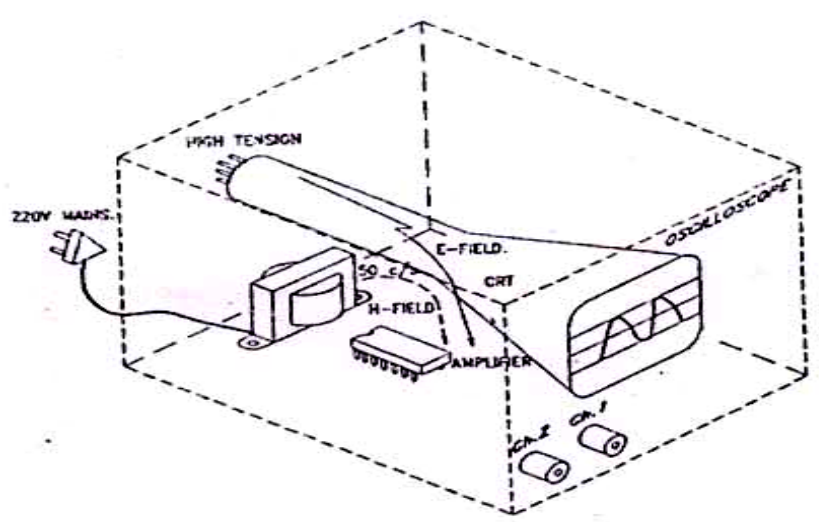

Figure-3. Example of internal EMI.

\section{TYPES OF NOISES}

Different types of noises occur in switching power supplies due to Electro Magnetic Interference. Some of them are Common Mode Noise, Differential Mode Noise, Non-Intrinsic Differential Mode Noise etc. These noises can be recognized by Spectrum Analyzer and they can be separated by a noise separator. In this paper, Common Mode Noise and Differential Mode noises are considered.

\section{A. Common Mode (CM) noise}

It is the noise between Phase and neutral at the supply side of the converter.

\section{B. Differential Mode (DM) noise}

It is the noise between two phases at the supply side of the converter.

\section{ELECTRO MAGNETIC INTERFERENCE (EMI) FILTER}

A novel topology of EMI Filter to suppress common mode and differential mode noises in switching power supplies is shown in Figure-4.

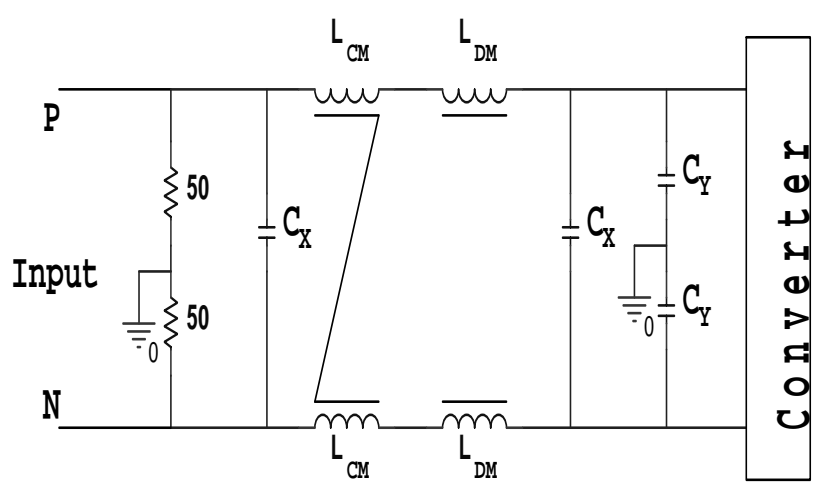

Figure-4. Proposed topology of EMI filter.

Here $\mathrm{L}_{\mathrm{CM}}$ and $\mathrm{C}_{\mathrm{Y}}$ can be used to suppress common mode noise. $L_{D M}$ and $C_{X}$ can be used to suppress differential mode noise. The combination of two 50 ohms resistors is called as Line Impedance Source Network (LISN). The EMI Filter and converter along with LISN can be connected to Spectrum Analyser through noise separator which separates CM noise and DM noise to observe the spectrums of total noise, CM noise and DM noise.

\section{DESIGN OF PROPOSED EMI FILTER}

It is extremely difficult to obtain an EMI filter design analytically. A practical approach was proposed in [8] to deal with the difficult issue. The approach was based on the following three conditions:

(i) Baseline (i.e. without filter) EMI noise for both CM and DM must be provided.

(ii) If the filter elements are properly arranged and sized, source impedance has little effect. Therefore, analytical design is possible without knowing exactly the source impedance values.

(iii) The focus of the design procedure is to meet the low frequency specification.

The design procedure of proposed EMI Filter is:

Step.1: Measure baseline EMI emission including total noise, CM noise and DM noise. For CM and DM noise measurement, a noise separator is needed.

Step.2: Determine attenuation requirements. The required attenuation is the discrepancy between the baseline noise and the EMI specs plus some correction factor. The correction factor has been discussed previously. It is “+3dB” for CM attenuation.

Step.3: Choose filter topology. This step also includes determining the order of the filter. Because of the nature of CM and DM noise source impedance, we don't have many choices on topology when the order is determined. 


$$
\begin{aligned}
& V_{C M, \text { req }}=V_{C M}-V_{\text {spec }}+3 d B \\
& V_{\text {DM,req }}=V_{\text {DM }}-V_{\text {spec }}+3 d B
\end{aligned}
$$

Step.4: Determine corner frequencies of noises. Using Spectrum analyzer and noise separator, the corner frequencies of CM Noise and DM Noise can be found as $f_{\mathrm{R}, \mathrm{CM}}$ and $f_{\mathrm{R}, \mathrm{DM}}$ respectively.

Step.5: Determine filter component values $\mathrm{L}_{\mathrm{CM}}$ and $\mathrm{L}_{\mathrm{DM}}$. After determining the filter corner frequency, the filter component values can be calculated using the equations given below.

$$
\begin{aligned}
& \text { For CM Noise, } \\
& \qquad \begin{aligned}
f_{\mathrm{R}, \mathrm{CM}}=1 /\left(2 \pi \sqrt{ }\left(2 \mathrm{Cy} \mathrm{L}_{\mathrm{CM}}\right)\right) \\
\mathrm{L}_{\text {leakage }}=0.5 \% \text { to } 2 \% \text { of } \mathrm{L}_{\mathrm{CM}}
\end{aligned}
\end{aligned}
$$

For DM Noise,

$$
\begin{aligned}
& f_{\mathrm{R}, \mathrm{DM}}=1 /\left(2 \pi \sqrt{ }\left(2 \mathrm{~L}_{\mathrm{D}} \mathrm{C}_{\mathrm{X}}\right)\right) \\
& \mathrm{L}_{\mathrm{DM}}=\left(\mathrm{L}_{\mathrm{D}}-\mathrm{L}_{\text {leakage }}\right) / 2
\end{aligned}
$$

Step.6: The final step is to measure the noise again with the designed filter.

\section{CONCLUSION}

In this paper, the issues of Electro Magnetic Interference (EMI) in switching power supplies are presented. Here, common mode noise and differential mode noise are considered. A novel procedure to design EMI Filter to mitigate EMI noise is proposed. This filter has the drawback that only CM noise and DM noise are considered. Different other noises can also be considered such as IDM, NIDM, mixed mode EMI noise etc.

\section{REFERENCES}

[1] J. P. Noon. 1994. A 250 kHz, 500W Power Factor Correction Circuit Employing Zero Voltage Transitions. Unitrode Power Supply Design Seminar Manual SEM1000.

[2] G. Moschopoulos, P. Jain and G. Joos. 1995. A novel zero voltage switched PWM boost converter. Proc., Power Electronics Specialists Conf. (PESC’95). pp. 694-700.

[3] Fu-Yuan Shih, Dan Y. Chen, Yan-Pei Wu and YieTone Chen. 1996. A Procedure for Designing EMI Filters for AC Line Applications. IEEE Transactions on Power Electronics. Vol. 11(1). January.

[4] Wei Zhang. 1998. Integrated EMI/Thermal design for Switching Power Supplies. M.S. Thesis. Virginia Polytechnic Institute and State University.

[5] Song Qu. 1999. Non Intrinsic Differential Mode Noise in Switching Power Supplies and its implications to EMI Filter design. M.S. Thesis. Virginia Polytechnic Institute and State University.

[6] Liyu Yang. 2003. Modeling and characterization of a PFC converter in the medium and high frequency ranges for predicting the conducted EMI. M.S. Thesis. Virginia Polytechnic Institute and State University. 